\title{
A Constructive Positive Realization with Sparse Matrices for a Continuous-Time Positive Linear System
}

\author{
Kyungsup Kim \\ Department of Computer Engineering, Chungnam National University, 99 Daehak-ro, Yuseong-gu, Daejeon 305-764, \\ Republic of Korea
}

Correspondence should be addressed to Kyungsup Kim; kskim@cse.cnu.ac.kr

Received 9 September 2013; Revised 22 November 2013; Accepted 10 December 2013

Academic Editor: Yong-Kui Chang

Copyright (C) 2013 Kyungsup Kim. This is an open access article distributed under the Creative Commons Attribution License, which permits unrestricted use, distribution, and reproduction in any medium, provided the original work is properly cited.

\begin{abstract}
This paper discusses a computational method to construct positive realizations with sparse matrices for continuous-time positive linear systems with multiple complex poles. To construct a positive realization of a continuous-time system, we use a Markov sequence similar to the impulse response sequence that is used in the discrete-time case. The existence of the proposed positive realization can be analyzed with the concept of a polyhedral convex cone. We provide a constructive algorithm to compute positive realizations with sparse matrices of some positive systems under certain conditions. A sufficient condition for the existence of a positive realization, under which the proposed constructive algorithm works well, is analyzed.
\end{abstract}

\section{Introduction}

We address the positive realization problem of a positive linear time-invariant system with a single input and a single output using the polyhedral convex cone introduced in [1]. Nonnegative constraints can be encountered in some applications, such as in probability theory [2], fiber-optic filters [3] and compartmental models in pharmacokinetics [4]. Several types of implementation of constructive methods of positive realizations for general transfer functions in discretetime have been proposed [5-8]. Most of the constructive methods that have been proposed to solve the positive realization problem in minimal dimensions have focused on discrete systems $[6,9,10]$. The positive realization of a continuous-time linear system can be obtained by finding a possibly minimal generator of polyhedral cone intervening reachability and observability. Finding a general minimal positive realization in a positive linear system is an open and difficult problem. Therefore, we instead try to find a positive realization of a positive system with a special structure and lower order by applying some constraints.

We note that constructive methods for positive realization in the continuous-time case have been studied less frequently than those in the discrete-time case. We will consider an efficient constructive method to compute positive realizations with sparse matrices for some given continuous-time positive systems with (possibly multiple) complex poles. We consider the relationship between positive realizations of the discrete-time systems introduced by $[10,11]$ and those of the continuous-time systems. A positive realization with sparse matrices is of practical interest, because it allows a simpler and cheaper realization in real system implementations. For example, we can construct the oriented weighted graphs that correspond to the computed positive realizations in applications such as circuits and fiber optic filters $[3,10,12]$. By reducing the number of incoming and outgoing branches of the weighted graph in a sparse positive realization, we can reduce the cost of the system implementation. In addition, because positive realizations are not unique, it is better to choose a sparse positive realization with fewer free parameters and a lower dimension. We also investigate the positive realization of a transfer function with multiple complex poles and present the sufficient conditions for the positive realization of this case.

In Section 2, we introduce the preliminary concepts for the analysis of a continuous-time positive linear system. In Section 3, we discuss the positive realization of a transfer function with simple poles. In Section 4, we consider 
a positive realization of a transfer function with multiple complex or real poles. We present the numerical results in Section 5 .

\section{Preliminary Concepts and Problem Formulation}

The notations used throughout the paper are introduced here. $\mathbb{R}$ is the set of real numbers $(-\infty, \infty)$, and $\mathbb{R}_{+}$is the set of positive real numbers. A convex cone $\mathscr{X}=\operatorname{cone}(X)$ is denoted by the smallest convex cone of a set $X$, which consists of all finite nonnegative linear combinations of elements of the set $X$. The dual cone, $\mathscr{X}^{*}$, of a cone $\mathscr{X}$ is defined by $\mathscr{X}^{*}=\left\{y \mid x^{T} y \geq 0, \forall x \in \mathscr{X}\right\}$. A convex cone $\mathscr{X}$ is said to be a polyhedral cone if $\mathscr{X}$ is spanned by a finite number of vector sets $X=\left\{x_{1}, \ldots, x_{m}\right\}$ such that $x_{i} \in \mathbb{R}^{n}$; that is, $\mathscr{X}=$ cone $\left(\left\{x_{1}, \ldots, x_{n}\right\}\right)$ and $X$ is a polyhedral generator of $\mathscr{X}$ [13]. Hereafter, $X$ is also denoted by the matrix with columns $x_{i} \in X$; that is, $X=\left[\begin{array}{llll}x_{1} & x_{2} & \ldots & x_{m}\end{array}\right]$, as long as it is not ambiguous. Given a general matrix $A=\left[a_{i j}\right]$, we write $A \geq 0$ if at least some entry $a_{i j} \geq 0$.

Consider a singleinput, single output linear time-invariant system of the form:

$$
\dot{x}(t)=A x(t)+B u(t), \quad y(t)=C x(t),
$$

where $A$ is an $n \times n$ matrix, $B$ is an $n \times 1$ matrix, and $C$ is an $1 \times n$ matrix. Define the transfer function $H(s)=C(s I-A)^{-1} B$ as the Laplace transform of the impulse response function $h(t)=$ $C \exp (A t) B$.

The linear system in (1) is a positive linear system if for any non-negative input $u(t)$ and non-negative initial state, the state trajectory $x(t)$, and the output $y(t)$ are always non-negative. We provide a formal statement of the positive realization problem for continuous-time positive systems. A matrix $A_{*} \in \mathbb{R}^{m \times m}$ is defined by a Metzler matrix if all of its off-diagonal elements are positive. A triple $\left(A_{*}, B_{+}, C_{+}\right)$is said to be a positive realization of the transfer function $H(s)$ if $A_{*}$ is a Metzler matrix, $B_{+} \geq 0$, and $C_{+} \geq 0$.

The positive realization problem consists of both the existence and the minimality problems. The existence problem is to find conditions and provide an algorithm for the existence of the positive realization. The minimality problem is to determine the minimal order allowed by the positive realization. It is known that in the absence of any nonnegative restriction, every proper rational function has a realization whose minimal dimension coincides with the McMillan degree [14]. There is no guarantee that properties such as existence and minimality hold for the positive realization problem $[1,9,15]$. The minimal order of the positive realization is generally larger than or equal to the McMillan degree. Our objective is to find a positive realization $\left(A_{*}, B_{+}, C_{+}\right)$ with the lower order from the given positive system $(A, B, C)$ in the absence of non-negative constraints.

Theorem 1 (see [1]). Let $\mathscr{P}$ be a polyhedral cone in $\mathbb{R}^{n}$ and $A \in \mathbb{R}^{n \times n}$. Thus, $\exp (A t) \mathscr{P} \subset \mathscr{P}$ for any $t \geq 0$ if and only if there is a $\lambda>0$ such that $(A+\eta I) \mathscr{P} \subset \mathscr{P}$ for all $\eta \geq \lambda$.
To prove the sufficiency of this problem, if $(A+\lambda I) \mathscr{P} \subset \mathscr{P}$ for some $\lambda,(A+\eta I) \mathscr{P}=(A+\lambda I) \mathscr{P}+(\eta-\lambda) \mathscr{P} \subset \mathscr{P}$ for all $\eta \geq \lambda$. Let $x_{k}=(t / k)(I+A k / t)^{k} x$. For some $t$ and $k / t \geq$ $\lambda, x_{k} \in \mathscr{P}$. Hence $\lim _{k \rightarrow \infty} x_{k}=\exp (A t) x \in \mathscr{P}$. Therefore, $\exp (A t) \mathscr{P} \subset \mathscr{P}$. To prove the necessity of this theorem, set $\mathbb{Q}=\mathscr{P}^{*}$. Let $P$ and $Q$ be polyhedral generators of $\mathscr{P}$ and $Q$, respectively. Let $\lambda_{i j}=-q_{i}^{T} A p_{j} / q_{i}^{T} p_{j}$ for $q_{i}^{T} A p_{j}<0$ and $\lambda_{i j}=$ 0 otherwise. Let $\lambda=\max \left(\lambda_{i j}\right)$. Thus, $(A+\lambda I) \mathscr{P} \subset \mathscr{P}$. The interested reader can refer to [1] for more details. We observe that if $(A+\eta I) \mathscr{P} \subset \mathscr{P}$ for a sufficiently large $\eta$, then there exists a positive matrix $A_{+} \geq 0$ such that $(A+\eta I) P=P A_{+}$. Therefore, we obtain a Metzler matrix $A_{*}=A_{+}-\lambda I$.

A reachable set $\mathscr{R}$ is the set of all points to which the states approach from the origin by non-negative inputs within finite time. It is wellknown that the reachable set consists of a closure of the non-negative linear combination set of the impulse response functions [1]. In Lemma 1 in [1], it was showed that $\mathscr{R}=\operatorname{cl}[\operatorname{cone}\{x \mid x=\exp (A t) B, t \geq 0\}]$, where $\operatorname{cl}(S)$ is a closure set of $S$. An observable set $\mathcal{S}$ is the set of initial states in which the output is non-negative for all $t \geq 0 . \mathcal{S}$ is defined by $\mathcal{S}=\{x \mid C \exp (A t) x \geq 0, \forall t \geq 0\}$. Assume that $x=\exp \left(A t_{0}\right) B \in \mathscr{R}$ for some $t_{0} \geq 0$. Because $C \exp (A t) x=C \exp \left(A\left(t+t_{0}\right)\right) B \geq 0, x \in \mathcal{S}$. Thus, we can verify that $\mathscr{R} \subset \mathcal{S}$. It is well-known that a positive realization problem of a given transfer function is reduced to the problem of finding an appropriate polyhedral cone in a room sandwiched by the reachability and observability cones $[1,9]$. The following theorem is presented as in [1] without proof.

Theorem 2 (see [1]). Let a transfer function $H(s)$ be a strictly proper rational function with degree $n$, whose (minimal) realization is given by $(A, B, C)$. Then, $H(s)$ has a positive realization $\left(A_{*}, B_{+}, C_{+}\right)$if and only if there exists a generator matrix $P$ and $\lambda \geq 0$ such that a polyhedral cone $\mathscr{P}=$ cone $(P)$ satisfies

(1) $(A+\lambda I) \mathscr{P} \subset \mathscr{P}$,

(2) $\mathscr{R} \subset \mathscr{P} \subset \mathcal{S}$,

where $P \in \mathbb{R}^{n \times m}, n \leq m, \mathscr{R}$ is a reachable set and $\mathcal{S}$ is an observable set.

From this theorem, $(A+\eta I) P=P A_{+}$and $\mathscr{R} \subset$ $\mathscr{P} \subset \mathcal{S}$ constitute necessary and sufficient conditions for the existence of a positive realization with a Metzler matrix $A_{*}=$ $A_{+}-\eta I$ in a continuous-time positive system. We note that the equation $(A+\eta I) P=P A_{+}$is related to the conditions of the existence of a positive realization in discrete time [ 9 , 10]. For the discrete-time case, several constructive methods to compute the polyhedral cones have been presented [6$8,10,11]$. We propose a constructive method to compute a positive realization with sparse matrices for a continuoustime positive system by modifying and extending the discrete version presented in [11].

Consider the relationship between the eigenvalues of $A$ in the state space realization and the poles of the transfer function $H(s)$. Let $\lambda(A)$ be an eigenvalue set of $A$. A dominant eigenvalue of $A$ is defined by a maximal real eigenvalue in 
$\lambda(A)$ and denoted by $\lambda_{0}$. The spectral radius of $A$ is denoted by $\rho(A)$; that is, $\rho(A)=\max |\lambda(A)|$. If $A$ is a Metzler matrix, then the dominant eigenvalue $\lambda_{0}$ of $A$ is a unique real eigenvalue and the real parts of the other eigenvalues of $A$ are less than $\lambda_{0}[16,17]$.

A positive system $H(s)$ is defined as positive realizable if we can find an equivalent positive realization of $H(s)$. For the continuous-time positive system, if $H(s)$ with realization $(A, B, C)$ is positive realizable, then we can find a positive realization $\left(A_{*}, B_{+}, C_{+}\right)$of the positive system $H(s)$. The transfer function of an impulse response function $h_{1}(t)=$ $h(t) \exp (\eta t)$ for all $t \geq 0$ is denoted by $\widetilde{H}(z)=C(z I-\widetilde{A})^{-1} B$ where $\widetilde{A}=A+\eta I$ and $z=s-\eta$. We note that $\widetilde{H}(z)$ has similar properties to the transfer function in the discrete-time domain. From Theorem 1 , if $(A, B, C)$ is positive realizable in the continuous-time domain, then $(A+\eta, B, C)$ is positive realizable in the discrete-time domain. Let $\mathbb{D}(r)$ be defined as a disk $\mathbb{D}(r)$ with a center 0 and a radius $r>0$. The function $\widetilde{H}(z)$ is analytical outside $\mathbb{D}(r)$ for properly large $r$. Therefore, $\widetilde{H}(z)$ is interpreted as a transfer function of a discrete-time domain on the disk $\mathbb{D}(r)$.

Set $A_{+}=\eta I+A_{*}$ and let $\widetilde{\lambda}_{k} \triangleq \lambda_{k}+\eta$ for each $k$ be an eigenvalue of $\widetilde{A}=\eta I+A$. The sequence $\left\{m_{i}\right\}$ of $\widetilde{H}(z)$ with $m_{i}=C \widetilde{A^{i-1}} B$ for all $i \geq 1$ is denoted by the Markov sequence introduced in [18, 19]. We can observe that $C \widetilde{A}^{i-1} B=C_{+} \widetilde{A}_{+}^{i-1} B_{+}$for all $i . \widetilde{H}(z)$ is described by $\widetilde{H}(z)=\sum_{k=0}^{\infty} m_{k} z^{-k}$. We note that the Markov sequence $\left\{m_{k}\right\}$ is similar to an impulse response sequence in the discretetime domain. We define a transfer function $H_{[L]}(z)$ that corresponds to the shifted Markov sequence $\left\{m_{k}\right\}_{k \geq L+1}$ (i.e., $\left.H_{[L]}(z)=\sum_{j=1}^{\infty} m_{j+L} z^{-j}\right)$ and $H^{c}(z)=\sum_{j=1}^{L} m_{j} z^{-j}$ for a given $L$.

Lemma 3. Assume that $\widetilde{H}(z)$ is decomposed into

$$
\widetilde{H}(z)=H^{c}(z)+z^{-L} H_{[L]}(z),
$$

and the L-shifted transfer function $H_{[L]}(z)$ has a positive realization $\left(A_{2}, B_{2}, C_{2}\right)$ with dimension $K$. Then, $\widetilde{H}(z)$ has a positive realization $\left(A_{+}, B_{+}, C_{+}\right)$with dimension $K+L$ such that

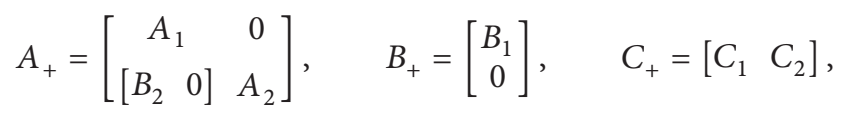

where the realization $\left(A_{1}, B_{1}, C_{1}\right)$ of $H^{c}(z)$ is given by

$$
\begin{aligned}
& A_{1}= {\left[\begin{array}{ccccc}
0 & 1 & 0 & \cdots & 0 \\
0 & 0 & 1 & \cdots & 0 \\
\vdots & & & \ddots & \vdots \\
0 & 0 & 0 & \cdots & 1 \\
0 & 0 & 0 & \cdots & 0
\end{array}\right], \quad B_{1}=\left[\begin{array}{c}
0 \\
0 \\
\vdots \\
0 \\
1
\end{array}\right], } \\
& C_{1}=\left[\begin{array}{lllll}
m_{L} & m_{L-1} & \cdots & m_{2} & m_{1}
\end{array}\right] .
\end{aligned}
$$

Proof. We observe that the transfer function $H^{c}(z)$ with a non-negative finite Markov sequence has an $L$-dimensional positive realization $\left(A_{1}, B_{1}, C_{1}\right)$. The positive realization of the transfer matrix $H_{[L]}(z)$ with dimension $K$ is given by $\left(A_{2}, B_{2}, C_{2}\right)$. The following combination of the two realizations yields a non-negative realization $(A, B, C)$ of $\widetilde{H}(z)$ :

$$
A=\left[\begin{array}{cc}
A_{1} & 0 \\
{\left[\begin{array}{ll}
B_{2} & 0
\end{array}\right]} & A_{2}
\end{array}\right], \quad B=\left[\begin{array}{c}
B_{1} \\
0
\end{array}\right], \quad C=\left[\begin{array}{ll}
C_{1} & C_{2}
\end{array}\right] .
$$

Therefore, $\widetilde{H}(z)$ has a positive realization of dimension $K+$ $L$.

A rational transfer function $\widetilde{H}(z)$ is primitive if the maximum modulus pole of $\widetilde{H}(z)$ is the only maximum real eigenvalue of $(A+\eta I)$, which is similar to concepts presented in discrete-time versions $[5,20]$. Thus, a transfer function $H(s)$ is defined by a primitive transfer function, if $\widetilde{H}(z)$ is a primitive transfer function for some $\eta$. Using partial fractional expansion of the rational transfer function $\widetilde{H}(z)$, we can define the primitive positive transfer function $\widetilde{H}(z)$ as

$$
\widetilde{H}(z)=\sum_{k=1}^{n_{0}} \frac{\beta_{k}^{(0)}}{\left(z-\tilde{\lambda}_{0}\right)^{k}}+\left\{\sum_{j=1}^{\tau} \sum_{k=1}^{n_{j}} \frac{\beta_{k}^{(j)}}{\left(z-\tilde{\lambda}_{j}\right)^{k}}\right\}
$$

where $n_{k}$ is the maximum number of the multiplicity of $\tilde{\lambda}_{k}=$ $\lambda_{k}+\eta$, which we call the Jordan-form realization. An $n_{j} \times n_{j}$ Jordan-form matrix $\widetilde{A}_{j}=J\left(\widetilde{\lambda}_{j}\right)$, an $n_{j} \times 1$ matrix $B_{i}$, and an $1 \times n_{j}$ matrix $c_{i}$ are given, respectively, as

$$
\begin{gathered}
J\left(\tilde{\lambda}_{j}\right) \triangleq\left[\begin{array}{ccccc}
\tilde{\lambda}_{j} & 1 & \cdots & 0 & 0 \\
0 & \tilde{\lambda}_{j} & \cdots & 0 & 0 \\
\vdots & & \vdots & \ddots & \vdots \\
0 & 0 & \cdots & \tilde{\lambda}_{j} & 1 \\
0 & 0 & \cdots & 0 & \tilde{\lambda}_{j}
\end{array}\right], \quad B_{j}=\mathbf{e}_{1, n_{j}}, \\
C_{j}=\left[\begin{array}{llll}
\beta_{n_{j}}^{(j)} & \beta_{n_{j}-1}^{(j)} & \cdots & \beta_{1}^{(j)}
\end{array}\right]
\end{gathered}
$$

for $1 \leq j \leq r$ [14]. A direct sum $A \oplus B$ is defined as the block matrix $A=\left[\begin{array}{ll}A & O \\ O & B\end{array}\right]$ with proper zero matrices $O$. The vector $\mathbf{e}_{i, n}$ represents a column vector of dimension $n$ with the $i$ th entry equal to 1 and all other entries equal to $0 . \widetilde{H}(z)$ has a canonical Jordan-form realization $(A, B, C)[14]$ such that

$$
\begin{gathered}
\widetilde{A}=\bigoplus_{j=0}^{\tau} \widetilde{A}_{i,} \quad B=\left[\begin{array}{lllll}
B_{0}^{T} & B_{1}^{T} & B_{2}^{T} & \cdots & B_{\tau}^{T}
\end{array}\right]^{T}, \\
C=\left[\begin{array}{lllll}
C_{0} & C_{1} & C_{2} & \cdots & C_{\tau}
\end{array}\right],
\end{gathered}
$$

where $A^{T}$ is the transpose matrix of $A$.

Theorem 4. A primitive transfer function $\widetilde{H}(z)$ is defined by (6). $\widetilde{H}(z)$ is decomposed into $\widetilde{H}(z)=H^{c}(z)+z^{-L} H_{[L]}(z)$. 
Assume that $\widetilde{H}(z)$ is positive realizable and $H_{[L]}(z)=F_{[L]}(z)+$ $G_{[L]}(z)$ such that $F_{[L]}(z)$ and $G_{[L]}(z)$ are defined by

$$
\begin{gathered}
F_{[L]}(z)=\sum_{k=2}^{n_{0}} \frac{\widehat{\beta}_{k}^{(0)}}{\left(z-\tilde{\lambda}_{0}\right)^{k}}, \\
G_{[L]}(z)=\sum_{j=1}^{\tau} \frac{\widehat{\beta}_{0, j}}{z-\widetilde{\lambda}_{0}}+\sum_{j=1}^{\tau} \sum_{i=1}^{n_{j}} \frac{\widehat{\beta}_{i}^{(j)}}{\left(z-\tilde{\lambda}_{j}\right)^{i}} .
\end{gathered}
$$

Then, a canonical Jordan-form realization of $H^{c}(z)$ is a positive realization, a canonical Jordan-form realization of $F_{[L]}(z)$ is a positive realization and $\widehat{\beta}_{0, j}>0$ satisfies

$$
\widehat{\beta}_{1}^{(0)}=\sum_{j=1}^{\tau} \widehat{\beta}_{0, j}, \quad \widehat{\beta}_{0, j} \geq \delta\left|\widehat{\beta}_{i}^{(j)}\right|
$$

for all $i$ with $1 \leq i \leq n_{j}$ and some $\delta>0$ for $G_{[L]}(z)$.

Proof. Because $\widetilde{H}(z)$ is positive realizable, we have the Markov parameter sequence $m_{k} \geq 0$. However, $\beta_{n_{0}}^{(0)}>0$ is necessary to obtain a positive Markov parameter sequence $m_{k} \geq 0$ (refer to [5]). The Jordan-form realization of $H^{c}(z)$ given by (4) is a positive realization and $H_{[L]}(z)$ is represented by $\left(\widetilde{A}, B, C \widetilde{A}^{L}\right)$. We have a realization $\left(A_{0}, B_{0}, C_{0} \widetilde{A}_{0}^{L}\right)$ of $F_{[L]}(z)$. Then $C_{0} \widetilde{A}_{0}^{L}=\left[\begin{array}{llll}\widehat{\beta}_{n_{0}}^{(0)} & \widehat{\beta}_{n_{j}-1}^{(0)} & \cdots & \widehat{\beta}_{1}^{(0)}\end{array}\right]$ is computed by

$$
\widehat{\beta}_{k}^{(0)}=\left(\begin{array}{c}
L \\
n_{0}-1
\end{array}\right) \beta_{n_{0}}^{(0)} \tilde{\lambda}_{0}^{L-n_{0}+1}+\sum_{i=0}^{n_{0}-2}\left(\begin{array}{c}
L \\
i
\end{array}\right) \beta_{i}^{(0)} \tilde{\lambda}_{0}^{L-i}
$$

where $\left(\begin{array}{l}n \\ k\end{array}\right)$ is the number of $k$-combinations from a given set with $n$ elements. For $\epsilon>0$, there is a sufficiently large $L$ such that $\left(\begin{array}{c}L \\ n_{0}-1\end{array}\right) /\left(\begin{array}{c}L \\ n_{0}-k\end{array}\right) \geq \epsilon\left(L-n_{0}+1\right)$ for all $k$ with $n_{0}>k \geq 0$. We can find a sufficiently large $L$ such that $\widehat{\beta}_{k}^{(0)}>0$. Therefore, the canonical Jordan-form realization of $F_{[L]}(z)$ is a positive realization. Because $\widehat{\beta}_{1}^{(0)}$ increases asymptotically as $L \rightarrow \infty$, we can choose a proper $L$ such that $\widehat{\beta}_{1}^{(0)}=\sum_{j=1}^{\tau} \widehat{\beta}_{0, j}$ for $\widehat{\beta}_{0, j}>$ $\delta\left|\widehat{\beta}_{i}^{(j)}\right|$ for all $i$ and $\widehat{\beta}_{0, j}>0$ for $G_{[L]}(z)$.

As $L$ increases, $\widehat{\beta}_{1}^{(0)}$ increases asymptotically. For sufficiently large $L$ and $\beta_{1}^{(0)}$, we obtain the transfer functions $G_{[L]}(z)$ in (10) with non-negative Markov sequences. Using Theorem $4, H^{c}(z)$ and $F_{[L]}(z)$ in (2) and (9), respectively, also have non-negative Markov sequences. Therefore, the positive realizations of $H^{c}(z)$ and $F_{[L]}(z)$ are obtained. A primitive function $G_{[L]}(z)$ with a simple dominant pole can be divided into

$$
G_{j}(z)=\frac{\widehat{\beta}_{0, j}}{z-\widetilde{\lambda}_{0}}+\sum_{i=1}^{n_{j}} \frac{\widehat{\beta}_{j}^{(i)}}{\left(z-\widetilde{\lambda}_{j}\right)^{i}} .
$$

Therefore, we need to find a positive realization for $G_{j}(z)$ for all $j$. In the continuous-time domain, we obtain $H(s)=\widetilde{H}(s-$ $\eta)=H^{c}(s-\eta)+(s-\eta)^{-L} H_{[L]}(s-\eta)$ by substituting $z=$ $s-\eta$. In the next section, we will discuss how to find a positive realization of $G_{j}(s-\eta)$ for each $j$.

\section{Positive Realizations for Systems with Simple Complex Poles}

Definition 5. Let $\mathfrak{P}_{m}(\rho)$ for $m \geq 1$ denote the set of points in the complex plane that lie in the interior of a regular polygon with $m$ edges with one vertex at point $\rho$ and a zero point at 0 . For $m \geq 2$, the polygon $\mathfrak{P}_{m}(\rho)$ is defined by a subset in $\mathbb{C}$ through the following inequalities:

$$
\mathfrak{P}_{m}(\rho)=\left\{x+i y \in \mathbb{C} \mid r \cos \left[\frac{(2 k+1) \pi}{m}-\varphi\right] \leq \rho \cos \frac{\pi}{m}\right\},
$$

for all $k$ with $0 \leq k \leq m$, where $x=r \cos \varphi$ and $y=r \sin \varphi$.

The complex number $x+i y$ corresponds to the point $(x, y)$ in the Cartesian plane $\mathbb{R}^{2}$. The polygon set $\mathfrak{P}_{m}(\rho)$ on the complex plane can be mapped onto $\mathbb{R}^{2}$. A $2 \times m$ matrix $\Psi$ that consists of the vertices of a polygon in the complex plane is defined as

$$
\Psi=\left[\begin{array}{ccccc}
1 & \cos \frac{2 \pi}{m} & \cos \frac{4 \pi}{m} & \cdots & \cos \frac{2 \pi(m-1)}{m} \\
0 & \sin \frac{2 \pi}{m} & \sin \frac{4 \pi}{m} & \cdots & \sin \frac{2 \pi(m-1)}{m}
\end{array}\right]
$$

for a given $m$. A cone generator matrix $P \in \mathbb{R}^{3 \times m}$ is defined as

$$
P=\left[\begin{array}{c}
\mathbf{E} \\
\Psi
\end{array}\right] \triangleq\left[\begin{array}{llll}
p_{1} & p_{2} & \cdots & p_{m}
\end{array}\right]
$$

where $\mathbf{E}$ represents an $1 \times m$ row vector with all entries equal to 1 ; the vectors $p_{i}$ are extreme points in a polyhedral cone, cone $(P)$. The real part of $\alpha$ is denoted by $\operatorname{Re}(\alpha)$ and the imaginary part of $\alpha$ is denoted by $\operatorname{Im}(\alpha)$. The angle of $\alpha$ is denoted by $\arg (\alpha)$. We consider a simple third-order positive proper transfer function $H(s)$ with a pair of strictly conjugate complex poles and a dominant real pole in partial fractional form such that

$$
H(s)=\frac{\beta^{(0)}}{s-\lambda_{0}}+\frac{\beta_{1}}{s-\lambda_{1}}+\frac{\bar{\beta}_{1}}{s-\bar{\lambda}_{1}},
$$

where $\lambda_{0}<0, \lambda_{1}$ is a complex pole with $\operatorname{Re}\left(\lambda_{1}\right)<\lambda_{0}, \bar{\lambda}_{1}$ is a complex conjugate of $\lambda_{1}$, and $\beta_{1}$ is complex number. $\mathrm{A}$ minimal real canonical realization of the transfer function is given by

$$
\begin{gathered}
A=\left[\begin{array}{ccc}
\lambda_{0} & 0 & 0 \\
0 & x & -y \\
0 & y & x
\end{array}\right] \triangleq \lambda_{0} \oplus \Gamma, \quad B=\left[\begin{array}{l}
1 \\
1 \\
0
\end{array}\right], \\
C=\left[\begin{array}{lll}
c_{1} & c_{2} & c_{3}
\end{array}\right],
\end{gathered}
$$

where $\oplus$ is a direct sum and $\lambda_{0}$ is a real pole with $\lambda_{0}<0$. A complex pole is defined as $\lambda_{1}=x+i y$ with $x<\lambda_{0}$ and another complex pole $\lambda_{2}$ is a conjugate of $\lambda_{1}$ (i.e., $\lambda_{2}=\bar{\lambda}_{1}$ ). Then, $\beta^{(0)}>0, c_{2}=2 \operatorname{Re}\left(\beta_{1}\right)$ and $c_{3}=2 \operatorname{Im}\left(\beta_{1}\right)$. 
Theorem 6. Assume that a transfer function $H(s)$ is defined by (17) where $\lambda_{0}>\operatorname{Re}\left(\lambda_{1}\right)$, and an extreme generator $P$ is constructed as described by (16). If

$$
\begin{gathered}
-\frac{\pi}{2}+\frac{\pi}{m} \leq \arg \left(\lambda_{1}-\lambda_{0}\right) \leq \frac{\pi}{2}-\frac{\pi}{m}, \\
\left|\beta_{1}\right| \leq \frac{\beta^{(0)}}{2},
\end{gathered}
$$

then there is a positive realization $\left(A_{*}, B_{+}, C_{+}\right)$of $H(s)$ with order $m$.

Proof. According to the assumption made in (19), the angle $\arg \left(\lambda_{1}-\lambda_{0}\right)$ is less than $(\pi / 2)-(\pi / m)$. As shown in Figure 1 , there is a sufficiently large positive real number $\eta>0$ such that the eigenvalues of $\eta I+A$ are located in $\mathfrak{P}_{m}\left(\lambda_{0}+\eta\right)$. $\mathfrak{P}_{m}\left(\lambda_{0}+\eta\right)$ is projected onto $\mathfrak{P}_{m}$ by dividing by $\lambda_{0}+\eta$. This means that the set $\left\{1,\left(\lambda_{1}+\eta\right) /\left(\lambda_{0}+\eta\right),\left(\bar{\lambda}_{1}+\eta\right) /\left(\lambda_{0}+\eta\right)\right\} \subset$ $\mathfrak{P}_{m}(1)$ is an eigenvalue set of $(\eta I+A) /\left(\lambda_{0}+\eta\right)$. As reported for the discrete-time cases in $[6,10]$, we can construct the polyhedral cone generator $P$ as described in (16) such that $(\eta I+A) /\left(\eta+\lambda_{0}\right) P \subset$ cone $(P)$. By Theorem $1,(\eta I+A) P=P A_{+}$ implies that $\exp (A t) P \subset \operatorname{cone}(P)$. We can demonstrate the invariance of $\exp (A t)$ for $P$. Therefore, we obtain a Metzler matrix $A_{*}=A_{+}-\eta I$.

We note that an invariant polytope argument is equal to an $\exp (A t)$-invariant polyhedral cone. We will show that $\mathscr{R} \subset \mathscr{P}$ and $\mathscr{P} \subset \mathcal{S}$. Because $B \in P$, there exists $B_{+}$ such that $P B_{+}=B$. It follows that $\mathscr{R} \subset \mathscr{P}$. We also know that $\left[\begin{array}{ll}c_{2} & c_{3}\end{array}\right]=\left[2 \operatorname{Re}\left(\beta_{1}\right) 2 \operatorname{Im}\left(\beta_{1}\right)\right]$. The inequality $C P \geq 0$ leads to the following sufficient condition: $\beta^{(0)} \mathbf{E}+$ $\left[2 \operatorname{Re}\left(\beta_{1}\right) 2 \operatorname{Im}\left(\beta_{1}\right)\right] \Psi \geq 0$. To satisfy the above inequality, we impose a sufficient condition such that $\left|\beta_{1}\right| \leq \beta^{(0)} / 2$ for sufficiently large $\beta^{(0)}$.

This result is similar to the original results of Dmitriev and Dynkin with respect to the Kolmogorov equation [21], which represents the earliest example of an invariant polytope argument. The invariance polytope approach, which is similar to the polyhedral convex cone, is adapted for a phase-type distribution [2]. If the conditions of Theorem 6 are satisfied, then we obtain a proper matrix $A_{+}$such that $(\eta I+A) P=P A_{+}$. However, the solutions for $A_{+}$are not unique. Furthermore, we wish to avoid a dense matrix for $A_{+}$for real system implementation. Therefore, we consider how to obtain a proper sparse matrix $A_{+}$with $2 n$ elements. The form of this realization matrix is related to circular Toeplitz matrices. Circular Toeplitz matrices have several useful properties (i.e., refer to [22]). For a given vector $\overrightarrow{\mathbf{t}}=\left(t_{0}, t_{1}, \ldots, t_{m-1}\right)$, a circular Toeplitz matrix $T(\overrightarrow{\mathbf{t}})$ is defined as

$$
T(\overrightarrow{\mathbf{t}})=\left[\begin{array}{ccccc}
t_{0} & t_{1} & t_{2} & \cdots & t_{m-1} \\
t_{m-1} & t_{0} & t_{1} & \cdots & t_{m-2} \\
t_{m-2} & t_{m-1} & t_{0} & \cdots & t_{m-3} \\
\vdots & \vdots & \vdots & \ddots & \vdots \\
t_{1} & t_{2} & t_{3} & \cdots & t_{0}
\end{array}\right],
$$

where each element $t_{i, j}$ is $t_{i, j}=t_{k}$ with $k=\bmod (j-i, m)$; here, $\bmod (n, m)$ is a residue of the integer $n$ modulo $m$.

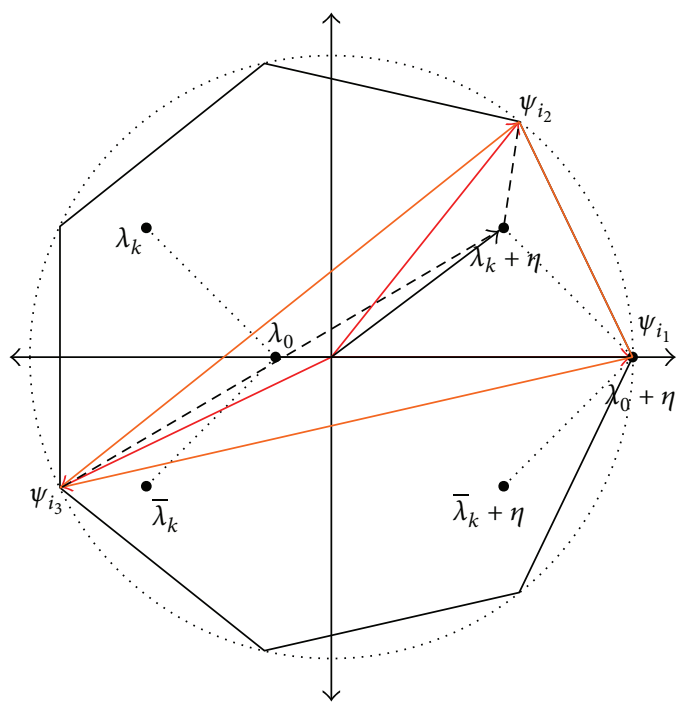

Figure 1: The geometric interpretation of $(\eta I+A) P \subset \mathscr{P}$ using $\mathfrak{P}_{m}(\rho)$.

Theorem 7. Assume that the conditions of Theorem 6 are satisfied. Then there exists a sparse positive circular Toeplitz matrix $A_{+} \geq 0$ with $3 m$ elements such that $(\eta I+A) P=P A_{+}$ for a proper $\eta>0$, and $A_{*}=A_{+}-\eta I$ is a Metzler matrix.

Proof. For $\epsilon=\lambda_{0}+\eta>0$, the eigenvalue set of $(A+$ $\eta I) / \epsilon$ is $\left\{1, z_{1}, \bar{z}_{1}\right\}$. Thus, $z_{1}, \bar{z}_{1} \in \mathfrak{P}_{m}$. We choose column vectors $\left\{\psi_{i_{1}}, \psi_{i_{2}}, \psi_{i_{3}}\right\}$ from $\Psi$ as in Figure 1 such that $z_{1} \in$ cone $\left\{\psi_{i_{1}}, \psi_{i_{2}}, \psi_{i_{3}}\right\}$. We also obtain $(A+\eta I) p_{1}=a_{1} p_{i_{1}}+a_{2} p_{i_{2}}+$ $a_{3} p_{i_{3}}$, where $\sum_{k} a_{k}=1$, with $a_{k} \geq 0$. Let $[n]$ be defined as $[n] \stackrel{\bmod }{=}(n-1, m)$. For $k \geq 1$,

$$
(A+\eta I) p_{1+k}=a_{1} p_{\left[i_{1}+k\right]}+a_{2} p_{\left[i_{2}+k\right]}+a_{3} p_{\left[i_{3}+k\right]} .
$$

We can obtain a sparse circular Toeplitz matrix $A_{+}=T(\overrightarrow{\mathbf{t}})$ such that a vector $\overrightarrow{\mathbf{t}}$ is defined by $t_{\left[i_{1}+k\right]}=a_{1}, t_{\left[i_{2}+k\right]}=a_{2}$, and $t_{\left[i_{3}+k\right]}=a_{3}$ and the other values of $t_{k}$ are zero. Thus, the matrix $A_{+}$is a sparse Toeplitz positive matrix.

\section{Positive Realizations for Systems with Multiple Poles}

Let us consider the properties of primitive transfer functions with a simple dominant pole and multiple complex poles (i.e., set $H(s)=G_{j}(s)$ for some $i$ in (13)). Let us consider a transfer function $H_{1}(s)$ with a single pair of multiple complex conjugate poles:

$$
H_{1}(s)=\sum_{k=1}^{n}\left\{\frac{\beta_{k}}{\left(s-\lambda_{1}\right)^{k}}+\frac{\bar{\beta}_{k}}{\left(s-\bar{\lambda}_{1}\right)^{k}}\right\},
$$

where the pole $\lambda_{1}$ and the coefficients $\beta_{i}$ are complex, and $\bar{\beta}_{i}$ is defined as the conjugate of $\beta_{i}$. The transfer function $H_{1}(s)$ 
with the partial fractional expansion in (23) has a canonical Jordan-form realization $(A, B, C)$ such that

$$
\begin{aligned}
& A=J\left(\lambda_{j}\right) \oplus J\left(\bar{\lambda}_{j}\right), \quad B=\left[\begin{array}{l}
\mathbf{e}_{n, n} \\
\mathbf{e}_{n, n}
\end{array}\right], \\
& C=\left[\begin{array}{lllllll}
\beta_{n} & \beta_{n-1} & \cdots & \beta_{1} & \bar{\beta}_{n} & \cdots & \bar{\beta}_{1}
\end{array}\right] .
\end{aligned}
$$

There exists a similarity transformation matrix $Q$ such that a block Jordan-form realization is given by

$$
\begin{aligned}
A_{1}=Q^{-1} A Q & =\left[\begin{array}{ccccc}
\Gamma_{0} & I & O & \cdots & O \\
O & \Gamma_{0} & I & \cdots & O \\
O & O & \Gamma_{0} & \cdots & O \\
\vdots & & & \ddots & \vdots \\
O & O & O & \cdots & \Gamma_{0}
\end{array}\right], \quad B_{1}=\left[\begin{array}{c}
0 \\
\vdots \\
0 \\
1 \\
1
\end{array}\right], \\
C_{1} & =\left[\begin{array}{lllll}
\beta_{n} & \bar{\beta}_{n} & \cdots & \beta_{1} & \bar{\beta}_{1}
\end{array}\right],
\end{aligned}
$$

where $\Gamma_{0}=\lambda_{1} \oplus \bar{\lambda}_{1}$. Let $\Delta=\left[\begin{array}{ll}1 & -i \\ 1 & i\end{array}\right]$. Using a block diagonal matrix $Q_{1}=w^{-n+1}\left(\bigoplus_{k=1}^{n} w^{k-1} \Delta\right)$ as a similarity transformation, we obtain a new real block Jordan-form realization $\left(J\left(\lambda_{1}, w\right), B_{2}, C_{3}\right)$ such that

$$
\begin{aligned}
& J\left(\lambda_{1}, w\right)=Q_{1}^{-1} A_{1} Q_{1}=\left[\begin{array}{ccccc}
\Gamma & w I & O & \cdots & O \\
O & \Gamma & w I & \cdots & O \\
O & O & \Gamma & \cdots & O \\
\vdots & & & \ddots & \vdots \\
O & O & O & \cdots & \Gamma
\end{array}\right], \\
& B_{2}=\left[\begin{array}{c}
0 \\
\vdots \\
0 \\
1 \\
0
\end{array}\right], \quad C_{2}=\left[\begin{array}{lllll}
\widetilde{\beta}_{2 n} & \tilde{\beta}_{2 n-1} & \cdots & \tilde{\beta}_{1}
\end{array}\right],
\end{aligned}
$$

where the entries of $C_{2}$ are defined by $\widetilde{\beta}_{2 k}=2 w^{k-n} \operatorname{Re}\left(\beta_{k}\right)$, $\widetilde{\beta}_{2 k-1}=2 w^{k-n} \operatorname{Im}\left(\beta_{k}\right)$ for each $k$ and $\Gamma=\left[\begin{array}{cc}x & -y \\ y & x\end{array}\right]$.

We consider a positive realization of transfer functions with a multiple complex conjugate pole pair. Let us define $\gamma_{m}(z)=\max \left\{\widehat{r} \mid(\widehat{r} \cos \theta, \widehat{r} \sin \theta) \in \mathfrak{P}_{m}, \widehat{r}>0\right\}$ for a given $z=r \cos \theta+i r \sin \theta$ and $m$. We propose a constructive method to generate a positive realization for a positive system with multiple poles, by modifying the discrete time version of [11], and provide the sufficient condition necessary for the proposed method in the next theorem.

Theorem 8. Assume that a positive system has a partial fractional expansion of the form

$$
H(s)=\frac{\beta^{(0)}}{s-\lambda_{0}}+H_{1}(s)
$$

with one dominant real pole $\lambda_{0}$ with a simple order and two complex poles $\lambda_{1}$ and $\bar{\lambda}_{1}$ with multiple orders $n \geq 1$, where $\beta^{(0)}>0, \operatorname{Re}\left(\lambda_{1}\right)<\lambda_{0}$ and $H_{1}(s)$ is defined as in (23). Set $z_{1}=\left(\eta+\lambda_{1}\right) /\left(\eta+\lambda_{0}\right)$ and $z_{2}=1 /\left(\eta+\lambda_{0}\right)$. One assumes that the following conditions are satisfied:

$$
\begin{gathered}
0<w \leq\left(1-\frac{\left|z_{1}\right|}{\gamma_{m}\left(z_{1}\right)}\right) \frac{\gamma_{m}\left(z_{2}\right)}{\left|z_{2}\right|}, \\
\left|\beta_{k}\right| \leq \frac{\beta^{(0)}}{(2 w)^{k-n}}
\end{gathered}
$$

for a given sufficiently large $\eta>0$ and all $1 \leq k \leq n$. Then, there exists a positive realization $\left(A_{*}, B_{+}, C_{+}\right)$of the transfer function $H(s)$ where $A_{*}$ is a Metzler matrix with order mn.

Proof. We obtain a real block Jordan-form realization $(A, B, C)$ of the transfer function $H(s)$ in (27) as follows:

$$
A=\lambda_{0} \oplus J\left(\lambda_{1}, w\right), \quad B=\left[\begin{array}{c}
1 \\
B_{2}
\end{array}\right], \quad C=\left[\begin{array}{ll}
1 & C_{2}
\end{array}\right],
$$

where $J\left(\lambda_{1}, w\right), B_{2}$, and $C_{2}$ are defined as in (26). We use the fact that there exists an $\eta>0$ such that there exists a $(\eta I+A)$-invariant cone $\mathscr{P}$ with $m n$ edges (i.e., $(\eta I+A) \mathscr{P} \subset \mathscr{P})$ to generalize the concept of the cone generator introduced in [10] for the case of multiple complex poles. To formulate a polyhedral cone generator with the $(\eta I+A)$-invariant property, a block shift matrix $Z \in \mathbb{R}^{2 n \times 2 n}$ and a matrix $K \in$ $\mathbb{R}^{2 n \times m}$ are defined as

$$
Z=\left[\begin{array}{ccccc}
O & O & \cdots & O & O \\
I & O & & O & O \\
O & I & \cdots & O & O \\
\vdots & \vdots & \ddots & \vdots & \vdots \\
O & O & \cdots & I & O
\end{array}\right], \quad K=\left[\begin{array}{c}
\Psi \\
O \\
O \\
\vdots \\
O
\end{array}\right]
$$

where $I$ is an identity matrix, $O$ is a zero matrix with the proper dimension, and the $2 \times m$ matrix $\Psi$ is defined as in (15). A cone generator matrix $P \in \mathbb{R}^{(2 n+1) \times m n}$ is defined as $P=\left[\begin{array}{llll}P_{1} & P_{2} & \cdots & P_{n}\end{array}\right]$, where $P_{j}$ is defined as $P_{j}=\left[\begin{array}{c}\mathbf{E} \\ \vartheta_{j}\end{array}\right]$ for $1 \leq$ $j \leq n-1$, such that $\mathrm{E}$ represents an $1 \times m$ row vector in which all entries equal 1 and $\vartheta_{j}=Z^{j-1} K$. The columns of matrix $P$ represent the extreme vertices of a finite generated cone $\mathscr{P}$ in $\mathbb{R}^{2 n+1}$ (i.e., cone $(P)=\mathscr{P}$ ) and are positive independent. The polyhedral cone $\mathscr{P}_{j}$ is generated by $P_{j}$, that is; $\mathscr{P}_{j}=\operatorname{cone}\left(P_{j}\right)$ for each $j$. We will prove that $\mathscr{P}$ is $A$-invariant (i.e., $A \mathscr{P} \subset \mathscr{P}$ ). Let $\mathscr{P}_{j}$ be the cone generated from the column vectors of $P_{j}$ (i.e., $\left.\mathscr{P}_{j}=\operatorname{cone}\left(P_{j}\right)\right)$. For $\lambda_{1} \in \mathfrak{P}_{m}\left(\lambda_{0}+\eta\right)$, we observe that $A P_{1} \in \mathscr{P}_{1}$. Note that $(A+\eta I) P_{j}$ for $j>1$ has nonzero values for the 1st, $j$ th, and $j+1$ th block components

$$
(A+\eta I) P_{j}=\underset{j}{j+1}\left(\begin{array}{c}
\mathbf{E} \\
\vdots \\
O \\
w \Psi \\
(\eta I+\Gamma) \Psi \\
O \\
\vdots
\end{array}\right) .
$$


The invariance property requires that $\left((A+\eta I) /\left(\lambda_{0}+\eta\right)\right) P_{j} \epsilon$ $\mathscr{P}$. Set $\widetilde{A}=(A+\eta I) /\left(\lambda_{0}+\eta\right)$. The matrix $\widetilde{A}$ has eigenvalues $\left\{1, z_{1}, \bar{z}_{1}\right\}$ with $\left|z_{1}\right|<1$. Thus, the positive realization problem with respect to $\widetilde{A}$ is closely related to that of the discrete-time cases reported in $[6,10]$. Choose $\left\{W_{j-1}, W_{j}\right\}$ such that

$$
\frac{(A+\eta I)}{\lambda_{0}+\eta} P_{j}=\alpha_{1} W_{j-1}+\alpha_{2} W_{j}
$$

for each $j$, where the values $\alpha_{j}$ satisfy $\alpha_{j} \geq 0, \alpha_{1}+\alpha_{2}=1$ and all of the entries in the first row of $W_{j} \in \mathscr{P}_{j}$ are equal to 1. A sufficient condition for a feasible solution $\left(\alpha_{1}, \alpha_{2}\right)$ should satisfy two inequalities, $w\left|z_{2}\right| / \gamma_{m}\left(z_{2}\right) \leq \alpha_{1}$ and $\left|z_{1}\right| / \gamma_{m}\left(z_{1}\right) \leq$ $\alpha_{2}$, and an equality $\alpha_{1}+\alpha_{2}=1$ for a given $w$. By rearranging the above conditions, we obtain the inequality $0<w \leq(1-$ $\left.\left|z_{1}\right| / \gamma_{m}\left(z_{1}\right)\right)\left(\gamma_{m}\left(z_{2}\right) /\left|z_{2}\right|\right)$. From this result, we observe that $w$ and $\eta$ are parameters that can be tuned to obtain a positive matrix. The polyhedral cone $\mathscr{P}$ is $\eta I+A$-invariant under the condition described by (28). We observe that $\mathscr{P}$, which is $\eta I+$ $A$-invariant, is equal to $\exp (A t) \mathscr{P} \subset \mathscr{P}$ by Theorem 1 . We obtain a sufficient condition as described by (28) to guarantee that $\mathscr{P}$ is $\exp (A t)$-invariant for all $t>0$. When the sufficient conditions are satisfied, there exists a positive $A_{+}$such that $(A+\eta I) P=P A_{+}$. Therefore, $A_{*}=A_{+}-\eta I$ is a Metzler matrix.

Next, let us consider $\mathscr{R} \subset \mathscr{P}$ and $\mathscr{P} \subset \mathcal{S}$. Because $B \in P$, it follows trivially that $\mathscr{R} \subset \mathscr{P}$. Finally, we will prove that $C=\left[\begin{array}{ll}\beta^{(0)} & C_{2}\end{array}\right] \in \mathscr{P}^{*}$. The entries $j_{1}=n-2 j+1$ and $j_{1}+1$ of $C_{2}$ are given by $\left[\begin{array}{ll}\widehat{c}_{j_{1}} & \widehat{c}_{j_{1}+1}\end{array}\right]=\left[\begin{array}{ll}\widetilde{\beta}_{2 j} & \widetilde{\beta}_{2 j-1}\end{array}\right](w)^{j-n}$, where $\widetilde{\beta}_{2 j-1}=$ $2 \operatorname{Im}\left(\beta_{i}\right)$ and $\widetilde{\beta}_{2 j}=2 \operatorname{Re}\left(\beta_{i}\right)$. An inequality $C P_{k} \geq 0$ for each $j \geq 1$ leads to the sufficient condition in (29) as follows:

$$
\beta^{(0)} \mathbf{E}+\left[2 \operatorname{Re}\left(\beta_{j}\right) 2 \operatorname{Im}\left(\beta_{j}\right)\right](w)^{j-n} \Psi \geq 0
$$

To satisfy the above inequality, we impose a sufficient condition such that $\left|\beta_{j}\right| \leq \beta^{(0)} /(2 w)^{j-n}$ for sufficiently large $\beta^{(0)}$ and each $1 \leq j \leq n$. We show that $C P_{j} \geq 0$. Therefore, there exists a positive realization $\left(A_{+}, B_{+}, C_{+}\right)$with order $n m$ such that $A_{+}=A+\eta I \in \mathbb{R}_{+}^{n m \times n m}, B_{+} \in \mathbb{R}_{+}^{n m}$ and $C_{+}^{T} \in \mathbb{R}_{+}^{n m}$ satisfy $(A+\eta I) P=P A_{+}, B=P B_{+}$and $C_{+}=C P$. Therefore, we obtain a Metzler matrix $A_{*}=A_{+}-\eta I$.

From Theorem 8 , we obtain a proper matrix $A_{+}$such that $(\eta I+A) P=P A_{+}$in the case of a transfer function with multiple poles and provide a sufficient condition for the existence of a positive realization. By the Previous theorem, we extended the positive realization problem to a continuoustime version based on the discrete-time version that was presented in [11]. As an additional contribution, we will show that a sparse positive realization can be constructed under the given conditions of Theorem 8 . In the next theorem, we show how to obtain a proper sparse matrix $A_{+}$with $3 m n$ elements.

Theorem 9. Assume that the conditions of Theorem 8 are satisfied. Then, there exists a sparse circular Toeplitz-type matrix $A_{+}$with at most $3 \mathrm{~nm}$ non-zero elements such that $(\eta I+A) P=P A_{+}$for a proper $\eta>0$. A sparse Toeplitz-type matrix $A_{+}$is defined by

$$
A_{+}=\left[\begin{array}{ccccc}
T_{1} & \widehat{\alpha}_{1} I & 0 & \cdots & 0 \\
0 & T_{2} & \widehat{\alpha}_{1} I & \cdots & 0 \\
0 & 0 & T_{2} & \cdots & 0 \\
\vdots & \vdots & \vdots & \ddots & \vdots \\
0 & 0 & 0 & \cdots & T_{2}
\end{array}\right],
$$

where $T_{1}=T\left(\overrightarrow{\mathbf{t}}_{1}\right), T_{2}=T\left(\overrightarrow{\mathbf{t}}_{2}\right)$, and $\widehat{\alpha}_{1}>0$. Vector $\overrightarrow{\mathbf{t}}_{1}$ has at most three non-zero elements and $\overrightarrow{\mathbf{t}}_{2}$ has at most two non-zero elements. Finally, one obtains a sparse Metzler matrix $A_{*}=$ $A_{+}-\eta I$.

Proof. Consider $(A+\eta I) p_{1,1}=a_{1} p_{1, i_{1}}+a_{2} p_{1, i_{2}}+a_{2} p_{1, i_{3}}$, where $p_{1, k}$ is the $k$ th-column of $P_{1}$. For sufficiently large $\eta$, we observe that a polyhedral convex cone $\mathscr{P}_{1}$ that includes $(A+\eta I) P_{1}$ can be obtained using a similar procedure to that outlined in Theorem 7. From (33), we note that, for $k>1$, $W_{k-1} \in \mathscr{P}_{k-1}$ is generated by the first column vector of $P_{k-1}$ and $W_{k} \in \mathscr{P}_{k}$ is generated by the convex combination of two column vectors of $P_{k}$. Then, $A P_{k}$ is positive linearly combined by the three vectors chosen from $P_{k}$ and $P_{k-1}$ in Theorem 8 . Therefore, we obtain a sparse Toeplitz-type matrix $A_{+}$as described by (35) and a Metzler matrix $A_{*}=A_{+}-\eta I$.

Let $N_{1}$ denote the sum of the orders of the (possibly multiple) non-negative poles in $\mathfrak{P}_{1}$, except for a dominant pole. Then the order of the positive realization is $N_{1}+1$. Let $N_{2}$ be the number of (possibly multiple) negative real poles in $\mathfrak{P}_{2}$, except for a dominant pole. Let $N_{m}(m \geq 3)$ denote the total number of complex conjugate pairs of (possibly multiple) complex poles of the transfer functions in the form $G_{k}(z)$ that belong to the region $\mathfrak{P}_{m}-\bigcup_{j=1}^{m-1} \mathfrak{P}_{j}$, except for a dominant pole. The order of the upper bound of the positive realization is bounded by $m N_{m}$ for each $m \geq 2$ by Theorem 8 . We obtain a positive realization of the primitive transfer function $H(z)$ with upper bound

$$
N=L+N_{0}+\left(1+N_{1}+\sum_{j \geq 2} j N_{j}\right) .
$$

If a transfer function $H(s)$ is positive realizable in a continuous-time linear system, then $H(s)$ is a primitive transfer function because the dominant eigenvalue of $A$ is real. Then, $\widetilde{H}(z)$ is primitive on $\mathbb{D}\left(\eta+\lambda_{0}\right)$ for a sufficiently large $\eta$, according to Theorem 1.

We now provide a summary of the process to construct the positive realization of a given transfer function $H(s)$ in a continuous-time domain.

(1) Reasonably, we assume that a primitive transfer function $\widetilde{H}(z)$ has a partial fractional expansion form as described by (6).

(i) Initially, a canonical Jordan-form realization $(A, B, C)$ of $H(s)$ is precomputed in the form presented in (8). 
(ii) Then, a minimum $\eta$ that maximizes $w$ and minimizes $m$ for all $\lambda_{i}, i \geq 1$ is computed to satisfy the condition in (28).

(2) Using Theorem 4, we choose a proper $L$ such that the realizations of $H^{c}(z)$ and $F_{[L]}(z)$ are positive realizations, and $G_{j}(z)$ for each $j$ satisfies the condition in (11), where $\widetilde{H}(z)=H^{c}(z)+s^{-L} H_{[L]}(z)$ and $H_{[L]}(z)=$ $F_{[L]}(z)+\sum_{j=1}^{\tau} G_{j}(z)$.

(i) A minimum $L$ is chosen such that $\widehat{\beta}_{k}^{(0)}>0$ for all $k$ and $\widehat{\beta}_{1}^{(0)}$ satisfies condition (11).

(ii) A positive realization of $H^{c}(z)$, which is denoted by $\left(\widehat{A}_{+}, \widehat{B}_{+}, \widehat{C}_{+}\right)$, is obtained by $(4)$.

(iii) A positive realization of $F_{[L]}(z)$ with order $N_{0}$, which is denoted by $\left(A_{0+}, B_{0+}, C_{0+}\right)$, is a canonical Jordan-form realization of $F_{[L]}(z)$ from Theorem 4 .

(3) We compute a positive realization of the primitive transfer function $G_{[L]}(s-\eta)$. For each $k$, we compute of the positive realization of $G_{k}(s-\eta)$ as follows.

(i) $\widehat{\beta}_{1}^{(0)}$ is properly divided into $\left\{\widehat{\beta}_{0, k}\right\}$ to satisfy the conditions in (11) and (29).

(ii) For each $\lambda_{k}$, a sparse positive realization of each primitive transfer function $G_{k}(s-\eta)$, which is denoted by $\left(A_{k+}, B_{+k}, C_{+k}\right)$, can be computed by Theorems 8 and 9 .

(4) Then a positive realization $\left(\bar{A}_{+}, \bar{B}_{+}, \bar{C}_{+}\right)$of the combination $F_{[L]}(z)+G_{[L]}(z)$ is integrated as

$$
\begin{gathered}
\breve{A}_{+}=\bigoplus_{k=0}^{\tau} A_{k+}, \quad \breve{B}_{+}=\left[\begin{array}{lll}
B_{0+}^{T} & \cdots & B_{\tau+}^{T}
\end{array}\right]^{T}, \\
\breve{C}_{+}=\left[\begin{array}{lll}
C_{0+} & \cdots & C_{\tau+}
\end{array}\right] .
\end{gathered}
$$

(5) A positive realization $\left(A_{+}, B_{+}, C_{+}\right)$of the transfer function $\widetilde{H}(z)$ is integrated using (3) of Lemma 3. The Metzler matrix $A_{*}=A_{+}-\eta I$ is set to obtain the positive realization $\left(A_{*}, B_{+}, C_{+}\right)$of $H(s)$.

\section{Numerical Results}

In this section, we present two typical examples to verify that the proposed method is able to compute the positive realizations of given transfer functions. In these examples, we used the MATLAB toolbox for numerical computations to generate the results. First, we consider the positive realization of a transfer function with simple complex poles.

Example 1. Consider the transfer function $H(s)$ of the form

$$
H(s)=\frac{1.5}{s+1}+\frac{0.25+0.25 j}{s+2-j}+\frac{0.25-0.25 j}{s+2+j}
$$

with poles equal to $-1,-2 \pm j$. In this example, $H(s)$ does not have a three-dimensional positive realization [1]. We observe that the poles of $H(s)$ are $\lambda_{0}=-1, \lambda_{1}=-2+j, \beta^{(0)}=1.5$, and $\beta^{(1)}=0.25+0.25 j$. Define a polyhedral generator $P$ as

$$
P=\left[\begin{array}{cccc}
1 & 1 & 1 & 1 \\
1 & 0 & -1 & 0 \\
0 & 1 & 0 & -1
\end{array}\right]
$$

From Theorem $7, \eta$ and $m$ are sufficiently large to generate a positive realization. We choose $\eta=2$ and $m=4$, which satisfies the condition of $(A+\eta I) P \subset \mathscr{P}$. Then, we can compute a positive realization $\left(A_{*}, B_{+}, C_{+}\right)$such that $A_{*}=$ $T(-2,0,0,1), B_{+}=\left[\begin{array}{llll}1 & 0 & 0 & 0\end{array}\right]^{t}$, and $C_{+}=\left[\begin{array}{llll}2 & 1 & 1 & 2\end{array}\right]$.

Example 2. Consider a primitive transfer function $H(s)$ with multiple poles. The eigenvalue set of $A$ is $\left\{\lambda_{0}, \lambda_{1}, \lambda_{2}\right\}$ such that $\lambda_{0}=-0.2, \lambda_{1}=-1.8+0.5 i$, and $\lambda_{2}=-2+0.3 i$. The multiple orders for the poles are $n_{0}=3, n_{1}=3$ and $n_{2}=3$. Using a canonical statespace realization of the transfer function, we can precompute a canonical Jordanform realization $(A, B, C)$, where $A=J\left(\lambda_{0}\right) \oplus J\left(\lambda_{1}\right) \oplus J\left(\bar{\lambda}_{1}\right) \oplus$ $J\left(\lambda_{2}\right) \oplus J\left(\bar{\lambda}_{1}\right)$, and $B$ and $C$ are defined in (8), where $C_{0}=$ $\left[\begin{array}{lll}3 & -1 & 1\end{array}\right], C_{1}=C_{2}=\left[\begin{array}{lll}1 & -1 & 0\end{array}\right]$, and $C_{3}=C_{4}=\left[\begin{array}{lll}-1 & 1 & -1\end{array}\right]$. Using Theorem 4 , we choose a proper number $L=2$ that satisfies the conditions to compute a realization $\left(\widehat{A}_{+}, \widehat{B}_{+}, \widehat{C}_{+}\right)$ of $H^{c}(z)$ such that $\widehat{A}_{+}=\left[\begin{array}{ll}0 & 1 \\ 0 & 0\end{array}\right], \widehat{B}_{+}=\left[\begin{array}{ll}0 & 1\end{array}\right]^{T}$, and $\widehat{C}_{+}=$ [3.0000 4.8000]. We observe that a canonical Jordan-form realization $\left(A_{0+}, B_{0+}, C_{0+}\right)$ of $F_{[L]}(z)$ in the form

$$
\begin{aligned}
A_{0+} & =\left[\begin{array}{ccc}
1.800 & 1.0000 & 0 \\
0 & 1.800 & 1.0000 \\
0 & 0 & 1.800
\end{array}\right], \\
C_{0+} & =\left[\begin{array}{lll}
3.2400 & 0.3600 & 0
\end{array}\right]
\end{aligned}
$$

and $B_{0+}=\mathbf{e}_{3,3}$ are positive-valued. We compute a positive realization $\left(A_{1+} \oplus A_{2+},\left[\begin{array}{l}B_{1+} \\ B_{2+}\end{array}\right],\left[\begin{array}{ll}C_{1+} & C_{2+}\end{array}\right]\right)$ of $G_{[L]}(z)$. We choose a proper $\eta$ and $w$ to satisfy conditions in (29) and (28), respectively. Therefore, we set a sufficiently large $\eta=2$ and choose $w_{1}=0.7340$ for $\lambda_{1}$ and $w_{2}=1.2804$ for $\lambda_{2}$ to obtain $\left(m_{1}, m_{2}\right)=(3,3)$. Using the proposed method, we obtain the positive matrix $A_{1+} \oplus A_{2+}$ with non-zero elements $3 m_{1} n_{1}+3 m_{2} n_{2}=54$. First, a $9 \times 9$ matrix $A_{1+}$ is computed as described by (35) with $T_{1}=T\left(\overrightarrow{\mathbf{t}}_{1}\right)$ and $T_{2}=T\left(\overrightarrow{\mathbf{t}}_{2}\right)$, where $\overrightarrow{\mathbf{t}}_{1}=\left[\begin{array}{lll}0.7333 & 0.2447 & 0.8220\end{array}\right], \overrightarrow{\mathbf{t}}_{2}=$ $\left[\begin{array}{lll}0.4887 & 0 & 0.5774\end{array}\right]$, and $\widehat{\alpha}_{1}=0.7340$. Then, $B_{1+}=\mathbf{e}_{9,9}$ and $C_{1+}=\left[\begin{array}{llll}3.3310 & 3.3310 & \cdots & 2.5554\end{array}\right]$. Another positive realization $\left(A_{2+}, B_{2+}, C_{2+}\right)$ can be obtained using similar methods. $\left(A_{+}, B_{+}, C_{+}\right)$can be obtained using (37) and (3). Therefore, we obtain a Metzler matrix $A_{*}=A_{+}-\eta I$ to obtain the positive realization $\left(A_{*}, B_{+}, C_{+}\right)$.

\section{Conclusion}

In this paper, we have presented a method to construct a positive realization with sparse matrices for a continuoustime positive system with complex multiple poles. In this 
approach, we use a constructive computational algorithm to obtain a positive realization with sparse matrices with the lowest order possible. We provided the conditions to construct a positive realization of sparse matrices with a bounded order for a positive continuous-time system. However, the positive realization is not guaranteed to be optimally minimal or sparse for a general positive system without any constraints. It remains an open problem to find sparse positive realizations with an optimal minimal dimension for general positive systems.

\section{Acknowledgments}

The author is indebted to the referee and Yong-Kui Chang for their careful work and useful suggestions, which improved the presentation of the results. This work was supported by research fund of Chungnam National University.

\section{References}

[1] Y. Ohta, H. Maeda, and S. Kodama, "Reachability, observability, and realizability of continuous-time positive systems," SIAM Journal on Control and Optimization, vol. 22, no. 2, pp. 171-180, 1984.

[2] C. A. O'Cinneide, "Phase-type distributions and invariant polytopes," Advances in Applied Probability, vol. 23, no. 3, pp. 515-535, 1991.

[3] L. Benvenuti and L. Farina, "The design of fiber-optic filters," Journal of Lightwave Technology, vol. 19, no. 9, pp. 1366-1375, 2001.

[4] R. F. Brown, "Compartmental system analysis: state of the art," IEEE Transactions on Biomedical Engineering, vol. 27, no. 1, pp. $1-11,1980$.

[5] B. Nagy and M. Matolcsi, "Algorithm for positive realization of transfer functions," IEEE Transactions on Circuits and Systems I, vol. 50, no. 5, pp. 699-702, 2003.

[6] B. Nagy, M. Matolcsi, and M. Szilvási, "Order bound for the realization of a combination of positive filters," IEEE Transactions on Automatic Control, vol. 52, no. 4, pp. 724-729, 2007.

[7] C. Hadjicostis, "Bounds on the size of minimal nonnegative realizations for discrete-time LTI systems," Systems and Control Letters, vol. 37, no. 1, pp. 39-43, 1999.

[8] B. Nagy and M. Matolcsi, "Minimal positive realizations of transfer functions with nonnegative multiple poles," IEEE Transactions on Automatic Control, vol. 50, no. 9, pp. 1447-1450, 2005.

[9] B. D. O. Anderson, M. Deistler, L. Farina, and L. Benvenuti, "Nonnegative realization of a linear system with nonnegative impulse response," IEEE Transactions on Circuits and Systems I, vol. 43, no. 2, pp. 134-142, 1996.

[10] L. Benvenuti, L. Farina, and B. D. O. Anderson, "Filtering through combination of positive filters," IEEE Transactions on Circuits and Systems I, vol. 46, no. 12, pp. 1431-1440, 1999.

[11] K. Kim, "A construction method for positive realizations with an order bound," Systems \& Control Letters, vol. 61, no. 7, pp. 759-765, 2012.

[12] L. Benvenuti and L. Farina, "Discrete-time filtering via charge routing networks," Signal Processing, vol. 49, no. 3, pp. 207-215, 1996.
[13] A. Berman and R. J. Plemmons, Nonnegative Matrices in the Mathematical Sciences, Society for Industrial and Applied Mathematics, Philadelphia, Pa, USA, 1994.

[14] T. Kailath, Linear Systems, Prentice Hall, New York, NY, USA, 1980.

[15] L. Farina, "On the existence of a positive realization," Systems and Control Letters, vol. 28, no. 4, pp. 219-226, 1996.

[16] N. K. Son and D. Hinrichsen, "Robust stability of positive continuous time systems," Numerical Functional Analysis and Optimization, vol. 17, no. 5-6, pp. 649-659, 1996.

[17] M. Valcher, "Reachability properties of continuous-time positive systems," IEEE Transactions on Automatic Control, vol. 54, no. 7, pp. 1586-1590, 2009.

[18] L. Farina, "Necessary conditions for positive realizability of continuous-time linear systems," Systems and Control Letters, vol. 25, no. 2, pp. 121-124, 1995.

[19] J. M. Van Den Hof, "Realization of continuous-time positive linear systems," Systems and Control Letters, vol. 31, no. 4, pp. 243-253, 1997.

[20] K.-H. Förster and B. Nagy, "Nonnegative realizations of matrix transfer functions," Linear Algebra and Its Applications, vol. 311, no. 1-3, pp. 107-129, 2000.

[21] Dmitriev and E. Dynkin, "On the characteristic numbers of a stochastic matrix," Doklady Akademii Nauk SSSR, vol. 49, pp. 159-162, 1945.

[22] R. Gray, “Toeplitz and circulant matrices: a review," Foundations and Trends in Communications and Information Theory, vol. 2, no. 3, pp. 155-239, 2006. 


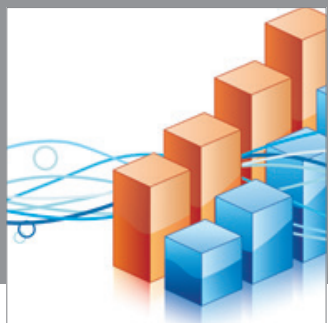

Advances in

Operations Research

mansans

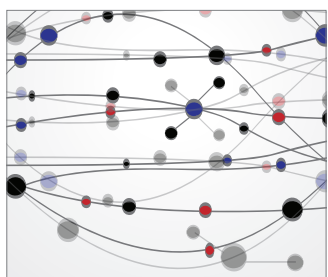

The Scientific World Journal
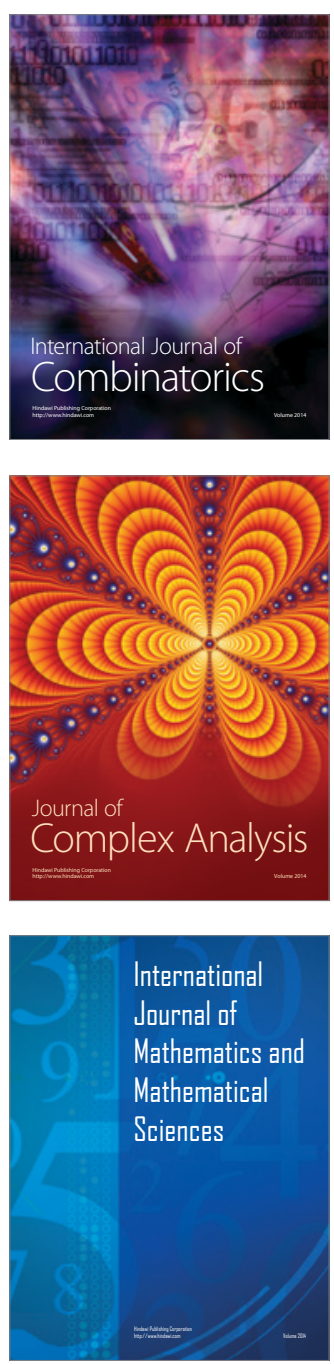
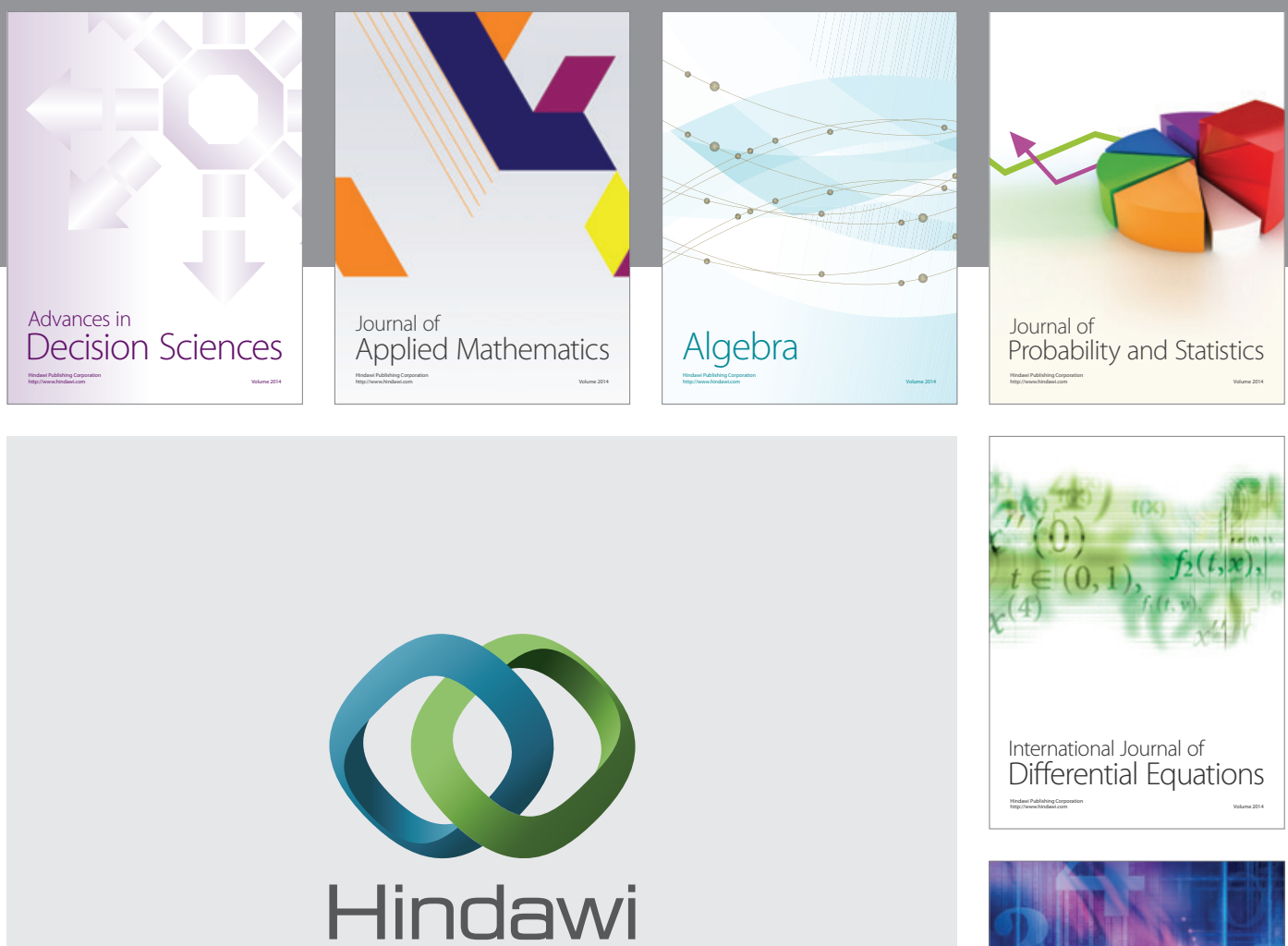

Submit your manuscripts at http://www.hindawi.com
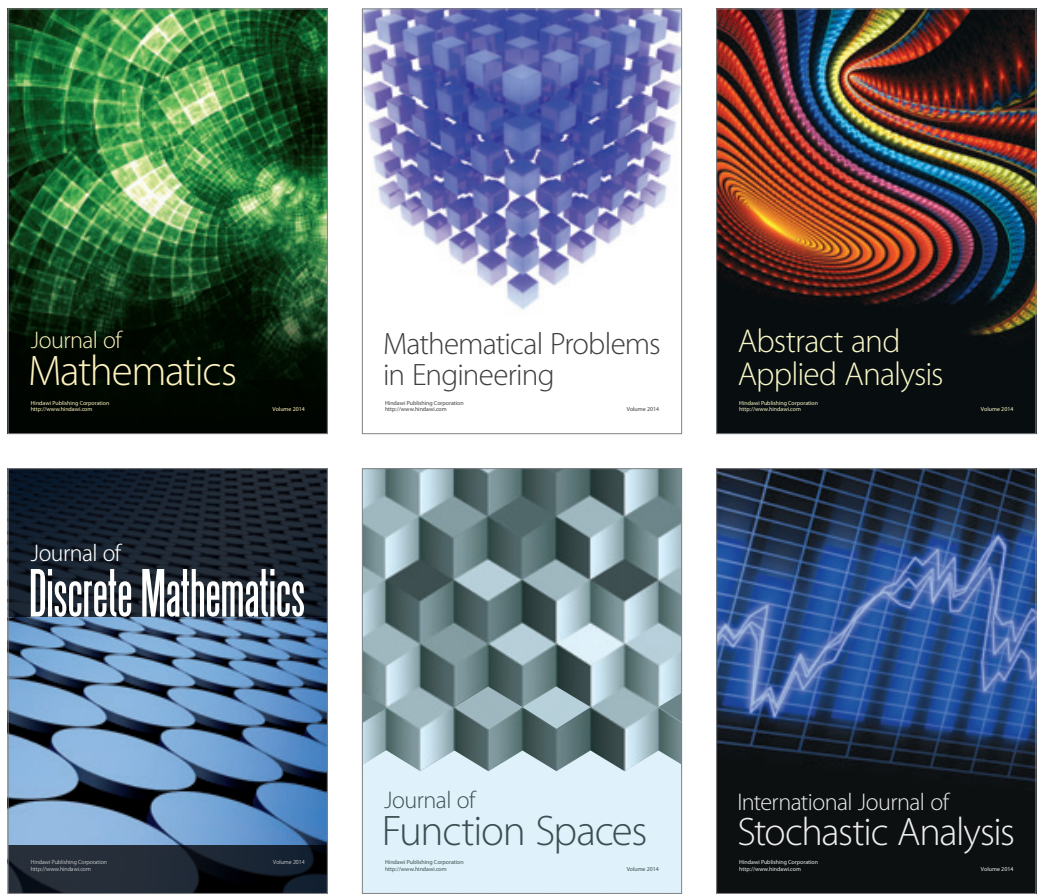

Journal of

Function Spaces

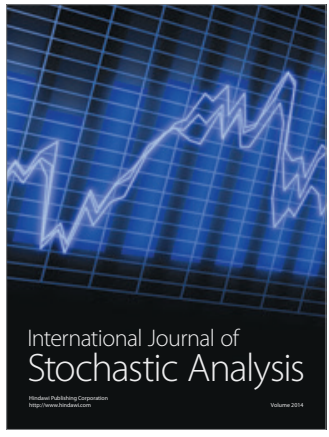

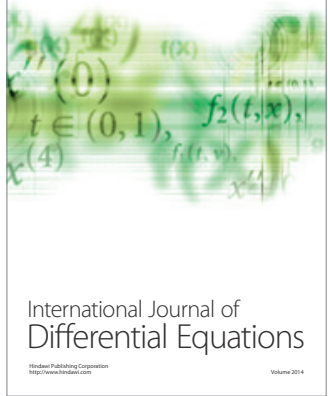
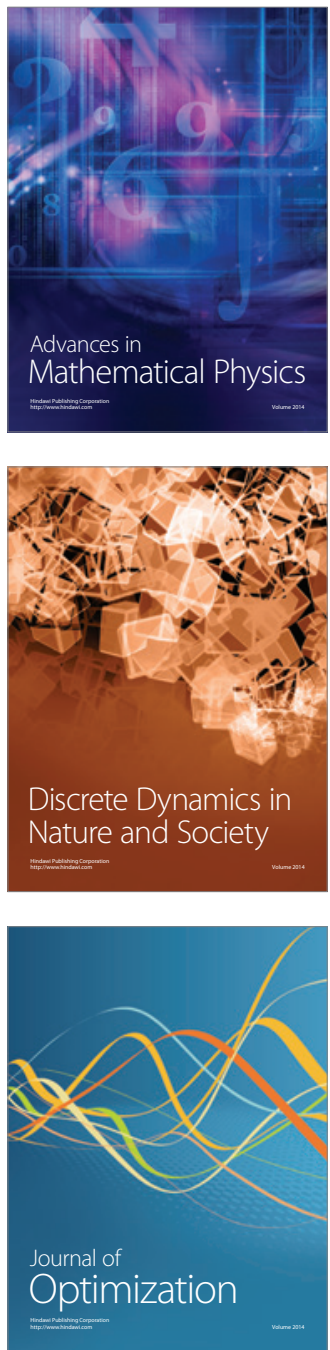